\title{
Biomateriales para Regeneración de Tejido Óseo Extraídos de Desechos de Pescado
}

\section{Biomaterials for Bone Tissue Regeneration Extracted from Fish Wastes}

\author{
D. Anaya-Barajas', J. Aguilar-Pliego², V. González-Vélez², M. Vélez-Tirado³ \\ 'UAM Azcapotzalco, Estudiante del Posgrado en Materiales, CBI \\ ¿UAM Azcapotzalco, Depto. Ciencias Básicas \\ ${ }^{3}$ Instituto de Catálisis y Petroleoquímica CSIC-UAM, Grupo de Biocatálisis
}

\section{RESUMEN}

Los biomateriales para regeneración ósea comúnmente se basan en el diseño natural del hueso, el cual mezcla la dureza de un material cerámico como la hidroxiapatita con la flexibilidad de un polímero como el colágeno. Ambos materiales pueden extraerse de los desechos del pescado consumido en la dieta humana, tales como escamas, piel e incluso huesos. Esta extracción tiene la ventaja de aportar biomateriales naturales, de baja toxicidad y como añadido, que evitan la transmisión de enfermedades que se ha reportado para colágenos provenientes de animales mamíferos. En este trabajo se hace una revisión de bibliografía referente a la extracción de colágeno tipo I, precursores de hidroxiapatita y síntesis de compósitos de ambos, para proponer la recuperación de estos biomateriales como una alternativa amigable con el ambiente y útil para usos médicos.

PALAbras Clave: Biomateriales; desechos de pescado; colágeno; hidroxiapatita 


\section{ABSTRACT}

Biomaterials for bone tissue regeneration are commonly based in the natural design of bone where the stiffness of a ceramic material as hydroxyapatite is combined with the flexibility of a polymer such as collagen. Both materials can be extracted from human consumed fish waste, such as scales, bones and skin. The recovery of these biomaterials from fish waste has the advantage of yielding natural materials of low toxicity, avoiding the possible disease transmission found for the collagen coming from mammalians. In this article, we review several reports about collagen type I and hydroxyapatite precursors extraction, and composite synthesis, from fish waste in order to propose these methods as a green alternative for biomaterials useful for medical purposes.

KEYWORDS: Biomaterials; fish wastes; collagen; hydroxyapatite

\section{Correspondencia}

DESTINATARIO: Daniela Ayala Barajas

INSTITUCIÓN: UAM Azcapotzalco

DIRECCIÓN: Av. San Pablo \#180, Col. Reynosa

Tamaulipas, Alcaldía Azcapotzalco, C. P. 02200,

Ciudad de México, México

CORREO ELECTRÓNICO: daniela_barajas@live.com

\section{Fecha de recepción:}

15 de junio de 2019

Fecha de aceptación:

15 de agosto de 2019 


\section{INTRODUCCIÓN}

El tejido óseo es un tejido del cuerpo que asemeja un material compósito ya que contiene una fase inorgánica que combina componentes minerales que contienen fosfato, calcio y grupos hidroxilos (hidroxiapatita) y una fase orgánica compuesta principalmente de colágeno. Esta útil combinación de materiales permite que el hueso tenga, al mismo tiempo, resistencia y flexibilidad ${ }^{[1]}{ }^{[2]}$.

La regeneración del tejido óseo se da de manera natural durante toda la vida, sin embargo, cuando existe una afección como fracturas, pérdida de masa ósea derivado de alguna enfermedad como tumores, degeneraciones o infecciones, donde el tejido ha sido destruido o removido lo cual llevan a que el hueso, en este caso, deje que cumplir la función vital de dar soporte y de permitir la comunicación bien protegida de la red nerviosa y la red sanguínea. Es necesario inducir o crear un tejido sustituto a través de la ingeniería de tejidos que cumpla las funciones vitales del tejido, esto es posible gracias a la compleja estructura a micro y nanoescala que presenta el hueso como material compósito bioactivo, mezcla de hidroxiapatita y colágeno ${ }^{[2]}{ }^{[3]}$.

Un biomaterial para regeneración ósea debe cumplir la función de reemplazar al hueso dañado al tiempo que permita que continúe la funcionalidad de los tejidos que forman parte de él, como vasos sanguíneos y nervios. Más aún, es deseable que sea bioactivo, es decir que induzca la reparación natural del tejido óseo conocida como osteogénesis. Los compósitos basados en hidroxiapatita (HAp) son muy utilizados, combinados con un polímero que les aporte flexibilidad y resistencia mecánica ${ }^{[1]}$.

La hidroxiapatita presente en los huesos tiene una composición similar a la HAp sintética, aunque se ha encontrado que esta última suele ser más frágil. Es un material cerámico que presenta una composición de
$\mathrm{Ca}_{10}\left(\mathrm{PO}_{4}\right)_{6}(\mathrm{OH})_{2}$ y mantiene una relación molar de calcio a fósforo $(\mathrm{Ca} / \mathrm{P})$ de 1.67. En el tejido óseo, la HAp está presente en un $60 \%$ en forma de nanocristales ${ }^{[3]}$. Para que la HAp sintética pueda ser empleada en aplicaciones médicas es necesario que cumpla con algunas normas, por ejemplo, debe cumplir un grado mínimo de cristalinidad del 45\% según la ISO 137779-2:2000, también debe ser ostoconductor, osteoinductivo y bioactivo ${ }^{[2][4]}$.

El colágeno (Col) es una de las principales proteínas estructurales del tejido conectivo de los vertebrados, constituye alrededor del $30 \%$ de sus proteínas totales ya que es el principal componente de tendones, piel, huesos, tejidos del sistema vascular entre otros tejidos ${ }^{[5]}$.

Existen 29 variantes reportadas de colágeno ${ }^{[6]}$, sin embargo, el colágeno tipo I es el componente principal de la matriz extracelular de mamíferos, contribuye a funciones fisiológicas únicas como la protección de tejidos y órganos, así como la regulación fisiológica del entorno celular para muchas especies animales [7]. Como característica principal del colágeno que está compuesto de una cadena de tres hélices, como unidad básica presenta las tres cadenas $\alpha$ que contienen una o más secuencias de polipéptidos (Gly-X-Y) y estas a su vez presentan entre 600 a 3,000 aminoácidos ${ }^{[1]}$.

El colágeno es ampliamente usado en las industrias cosmetológicas, biomédicas, farmacéutica por su excelente biocompatibilidad, biodegradabilidad y baja antigenicidad ${ }^{[8]}$, actualmente se ha extraído de piel y huesos de animales bovinos y porcinos. Sin embargo, ambos han presentado brotes de encefalopatía espongiforme bovina y encefalopatía espongiforme transmisible, es por ello que se pretende extraer el colágeno de animales que no tengan la capacidad de transmitir alguna enfermedad, por lo tanto, se ha optado por la obtención del colágeno a partir de animales marinos, ya que el colágeno tipo I es más común en piel, escamas, huesos y aletas de peces ${ }^{[9]}$. 
El presente artículo de revisión tiene como propósito mostrar los trabajos que se han reportado respecto a la extracción de HAp y de colágeno a partir de desechos de pescado, ya que esta recuperación puede ser una alternativa prometedora y amigable con el ambiente para obtener precursores de biomateriales compósitos para regeneración ósea.

\section{CRONOLOGÍA}

\section{Colágeno}

En los años noventas Kimura y colaboradores aislaron colágeno equinodermos indicando la presencia de colágeno fibrilar en la pared del cuerpo ${ }^{[10]}$. Unos años después Nomura y colaboradores extrajeron colágeno de escamas de sardina, obteniendo un rendimiento del $5 \%$. En este estudio se propone que la etapa de desmineralización de las escamas es un paso importante para la recuperación del colágeno ${ }^{[7]}$.

En el año 2000, Nagai y colaboradores ${ }^{[1]}$ aislaron colágeno a partir de la piel, las aletas y los huesos del pescado. En su trabajo hacen mención de la escasa información disponible sobre la presencia de colágeno en tejidos calcificados de peces. Determinan que la temperatura de desnaturalización del colágeno obtenido de la piel de las diferentes especies de peces es de alrededor de $25^{\circ} \mathrm{C}, 10^{\circ} \mathrm{C}$ menos que el colágeno porcino, mientras que para el colágeno extraído de hueso de pescados es de $29^{\circ} \mathrm{C}$. Más tarde, varios trabajos siguen la metodología de Nagai para extraer colágeno soluble en ácido y en pepsina a partir de escamas ${ }^{[5]}\left[{ }^{[12]}\right.$, de entre los cuales destaca Jongjareonrak y colaboradores porque reporta rendimientos del 9 y $4.7 \%$ para colágenos solubles en ácido y en pepsina, respectivamente ${ }^{[5]}$. Posteriormente Dasong y colaboradores en 2012 extrajeron y caracterizaron colágeno soluble en pepsina obtenido de escamas, aletas, huesos y piel. Su trabajo es relevante porque describe detalladamente la metodología que siguieron, mencionando las diferencias de solubilidad en las reacciones de extracción del colágeno obtenido de escama y hueso contra el obtenido de piel. Dos años después Dasong compara el colágeno soluble en ácido obtenido de la piel y escamas de cuatro tipos de carpas, observando diferencias en la presencia y concentración de las cadenas $\alpha$, mostrando tres cadenas $\alpha 1, \alpha 2$ y $\alpha 3$, y no solo dos como se había reportado anteriormente ${ }^{[8]}$.

Más recientemente Chuaychan y colaboradores aislaron colágeno soluble en pepsina (PSC) y colágeno soluble en ácido (ASC) a partir de escamas y siguiendo la metodología establecida por Nagai; sin embargo, reportan rendimientos mucho menores (0.38\% ASC, $1.06 \%$ PSC) ${ }^{[9]}$. Wang y colaboradores logran extraer colágeno tipo I soluble en ácido y en pepsina con rendimientos de alrededor del $20 \%$ de piel de lochas nativas de Asia, postulando a este procedimiento como una buena alternativa al colágeno extraído de mamíferos. Finalmente Bhuimbar y colaboradores reportaron la extracción de colágeno soluble en ácido láctico proveniente de la piel de la medusa y muestran su potencial, junto con quitosano, como antibacterial ${ }^{[13]}$.

\section{Hidroxiapatita}

Wen-Kuang Liu et al. extrajeron hidroxiapatita a partir de escamas para emplearla en la remoción de iones de plomo en aguas residuales ${ }^{[14]}$. Ulfyana y su equipo aislaron hidroxiapatita de las escamas de pescado reportando un rendimiento de la obtención de calcio de $83.62 \%$ y $12.7 \%$ de fosfato. Contemplaron que la HAp debía cumplir un grado de cristalinidad mínimo del $45 \%$ para poder ser un material empleado en el área de los biomateriales, realmente de la escama sólo obtuvieron el precursor de HAp que es el óxido de calcio $(\mathrm{CaO})$, para obtener la hidroxiapatita se añadieron ácido fosfórico e hidróxido de amonio ${ }^{[4]}$. Más recientemente, Wibison y colaboradores describen la obtención de precursores de un material con fases secundarias de $\beta$-TCP para la HAp de las escamas, éste estudio lo realizaron a partir de escamas de nemátodos y conchas de cangrejo por métodos húmedos ${ }^{[15]}$. 


\section{Compósitos HAp/colágeno}

Sasmal y Begam sintetizaron un material compósito de hidroxiapatita/colágeno, extrajeron el colágeno a partir de piel de un pescado y posteriormente elaboraron el compósito por método químico húmedo ${ }^{[16]}$. Yoruc y colaboradores sintetizaron el compósito de HAp/col, utilizando colágeno tipo I de origen bovino y los precursores de HAp a través del método de precipitación ${ }^{[17]}$. Posteriormente Yoruc y colaboradores sintetizaron un compósito de HAp/ $\beta$-fosfato tricalcio/colágeno (HAp/ $\beta-\mathrm{TCP} / \mathrm{Col})$, ellos aislaron el fosfato de calcio y el colágeno de escamas obteniendo una concentración en porcentaje de peso para el calcio igual a 31.8\% y $20 \%$ en peso del fosfato ${ }^{[18]}$. Recientemente Chai y colaboradores reportaron la comparativa de dos procesos de elaboración de estructuras híbridas de colágeno/ hidroxiapatita, en uno de los experimentos colocaron moléculas de colágeno (Col-M/HAp) y en el otro colocaron fibras de colágeno (Col-F/HAp), con el objetivo de conocer los procesos de auto organización de las estructuras híbridas con la finalidad de comprender mejor el mecanismo de formación de los tejidos óseos ${ }^{[19]}$.

\section{MÉTODOS}

\section{Extracción de colágeno a partir de piel de pescado}

Para obtener colágeno soluble en ácido y soluble en pepsina, Nagai y colaboradores establecieron las bases de la metodología que se continúa empleando en estudios recientes (Figura 1). Hacen énfasis en mantener la piel a $4^{\circ} \mathrm{C}$ durante la extracción para evitar desnaturalizar el colágeno. Proponen un tratamiento previo de cortado de la piel y lavado, a continuación la hidrólisis o remoción de proteínas no colagenosas y la eliminación de grasas suspendiendo los residuos anteriores en una solución de alcohol butílico. Después, efectúan el tratamiento ácido para obtener colágeno soluble en ácido, o en una solución de ácido más pepsina para obtener colágeno soluble en pepsina. Luego de precipitar, realizan la diálisis y la liofilización del colágeno ${ }^{[11]}$.

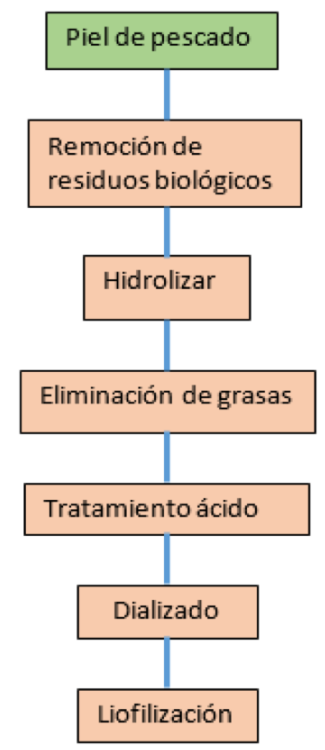

FIGURA 1. Etapas para la extracción de colágeno a partir de piel de pescado.

\section{Extracción de colágeno a partir de escamas}

La extracción de colágeno a partir de las escamas de pescado ha sido reportada por Dasong y colaboradores, cuya metodología ha sido citada por trabajos más recientes (Figura 2).

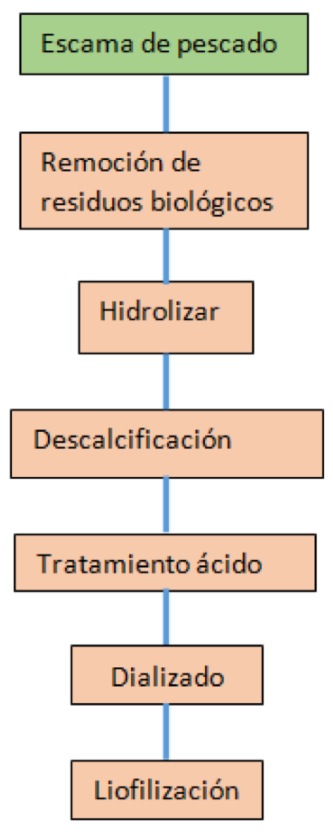

FIGURA 2. Etapas para la extracción de colágeno a partir de escamas de pescado. 
Este grupo propone seguir los mismos pasos reportados por Nagai ${ }^{[11]}$ para la extracción de colágeno a partir de piel, incorporándoles algunas modificaciones en la concentración de las soluciones y sustituyendo la eliminación de grasa en piel por la descalcificación de escamas ${ }^{[20]}$.

\section{Extracción de HAp a partir de escamas}

Ulfyana y colaboradores han descrito recientemente una metodología para extraer precursores de HAp de las escamas del pescado (Figura 3). En ella incluyen como primer paso la remoción de todo residuo biológico no deseado, para posteriormente ser sometidas a hidrólisis, esterilización, secado y calcinación.

Con ello, se obtiene el óxido de calcio como precursor de la HAp. Posteriormente vierten un una solución el óxido de calcio con el precursor de fosfato (ácido fosfórico), una vez mezclados estos componentes, se continúa con los tratamientos mecánico y térmico que permiten obtener la HAp ${ }^{[4]}$.

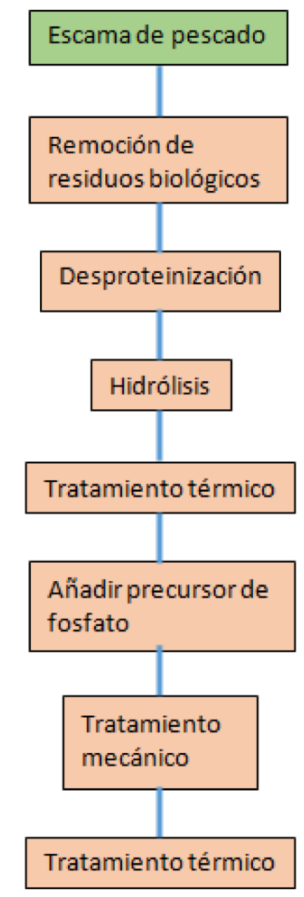

FIGURA 3. Etapas para la extracción de HAp a partir de escamas de pescado.

\section{Síntesis de compósito HAp/Col}

En cuanto a la síntesis del material compósito (Figura 4), Sasmal y Begam propusieron la obtención del mismo a través del método de precipitación que consiste en mezclar el colágeno con el precursor de fosfato y por adición incorporar el calcio hidratado, posteriormente dejar envejecer el material, filtrar y liofiizarlo ${ }^{[16]}$.

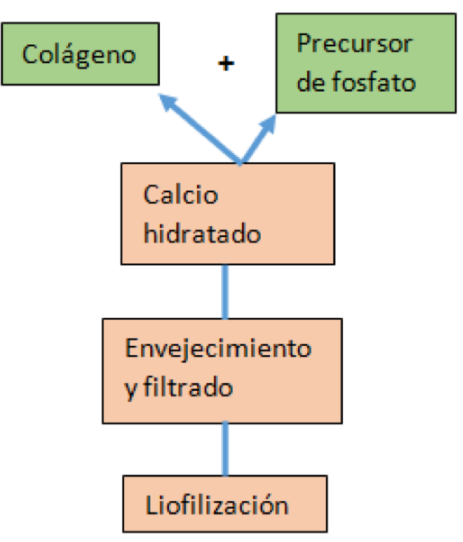

FIGURA 4. Etapas para la síntesis del compósito HAp/Col.

\section{Caracterización y cuantificación de colágeno extraído}

Para confirmar la presencia de colágeno tipo I en el material extraído de piel o escamas, como se describió en las Figuras 1 y 2, se suelen utilizar técnicas de caracterización fisicoquímica tales como Electroforesis de Gel (para determinar qué tipo de colágeno se tiene), espectroscopía infrarroja con transformada de Fourier (FT-IR) (para ver los enlaces característicos del material en cuestión), espectrofotometría UV-visible (verificar la presencia del compuesto/material) y BET (determinación de área superficial, porosidad del material). Todas estas técnicas detectan la presencia de las cadenas $\alpha 1$ y $\alpha 2$, y de las bandas amida que son características del colágeno tipo I ${ }^{[6]}{ }^{[8]}$.

En cuanto a la caracterización de la HAp se emplean técnicas fisicoquímicas como difracción de rayos-x (XRD) para determinar la estructura cristalina y con ello el grado de cristalinidad, FT-IR para identificar los grupos funcionales característicos de la HAp, microsco- 
pía electrónica de barrido (SEM) para analizar la morfología del material, espectrometría de energía dispersiva (EDS) para cuantificar la presencia de los elementos presentes en la muestra, entre otras técnicas ${ }^{[21]}$.

TABLA 1. Rendimiento de la extracción de colágeno.

\begin{tabular}{|c|c|c|c|c|c|}
\hline \multirow{3}{*}{$\begin{array}{c}\text { REG. } \\
\text { Asia }\end{array}$} & \multirow{2}{*}{\multicolumn{2}{|c|}{ PESCADO }} & \multicolumn{2}{|c|}{ REND. \% } & \multirow{3}{*}{$\begin{array}{c}\text { REF. } \\
{[7]}\end{array}$} \\
\hline & & & \multirow{2}{*}{$\begin{array}{c}\text { ASC } \\
--\end{array}$} & \multirow{2}{*}{$\begin{array}{c}\text { PSC } \\
-- \\
\end{array}$} & \\
\hline & Sardina & Escama & & & \\
\hline Asia & $\begin{array}{l}\text { Lateolabrax } \\
\text { japonicus } \\
\text { (Lubina japonesa) }\end{array}$ & $\begin{array}{l}\text { Piel } \\
\text { Huesos } \\
\text { Aletas }\end{array}$ & $\begin{array}{c}51.4 \\
40.7 \\
5.2\end{array}$ & $\begin{array}{l}-- \\
-- \\
--\end{array}$ & \multirow{3}{*}[11]{} \\
\hline Asia & $\begin{array}{l}\text { Scomber japonicus } \\
\text { (Estornino) }\end{array}$ & Piel & 49.8 & -- & \\
\hline Asia & $\begin{array}{l}\text { Heterodontus } \\
\text { (Tiburón toro o } \\
\text { bullhead shark) }\end{array}$ & Piel & 50.1 & -- & \\
\hline Asia & $\begin{array}{l}\text { Katsuwonus } \\
\text { pelamis } \\
\text { (Listado) }\end{array}$ & Hueso & 42.3 & & {$[11]$} \\
\hline Asia & $\begin{array}{l}\text { Pagrus major } \\
\text { (Pargo japonés) }\end{array}$ & Escama & -- & \multirow[b]{2}{*}{2} & \multirow[b]{2}{*}[12]{} \\
\hline Asia & $\begin{array}{l}\text { Oreochromis } \\
\text { niloticus } \\
\text { (Tilapia del Nilo) }\end{array}$ & Escama & -- & & \\
\hline Asia & Sardina & Escama & -- & 50.9 & \multirow{3}{*}[22]{} \\
\hline Asia & $\begin{array}{l}\text { Lateolabrax } \\
\text { japonicus } \\
\text { (Lubina japonesa) }\end{array}$ & Escama & -- & 41 & \\
\hline Asia & Pagellus bogaraveo & Escama & -- & 37.5 & \\
\hline Asia & $\begin{array}{l}\text { Lutjanus vitta } \\
\text { (Pargo) }\end{array}$ & Piel & 9 & 4.7 & {$[5]$} \\
\hline Asia & $\begin{array}{l}\text { Siganus fuscescens } \\
\text { (Pez conejo) }\end{array}$ & Piel & 3.6 & -- & \multirow{5}{*}{ [23] } \\
\hline Asia & $\begin{array}{l}\text { Kyphosus bigibbus } \\
\text { (Carpa marrón) }\end{array}$ & Piel & 3.6 & -- & \\
\hline Asia & Myliobatis tobijei & Piel & \multirow{3}{*}{$\begin{array}{l}3.5 \\
5.7\end{array}$} & \multirow{3}{*}{-- } & \\
\hline Asia & Dasyatis akajei & Piel & & & \\
\hline Asia & Dasyatis laevigata & Piel & & & \\
\hline Europa & $\begin{array}{l}\text { Cyprinus carpio } \\
\text { (Carpa) }\end{array}$ & $\begin{array}{l}\text { Piel } \\
\text { Escama } \\
\text { Hueso }\end{array}$ & $\begin{array}{l}41.3 \\
1.35 \\
1.06\end{array}$ & $\begin{array}{l}-- \\
-- \\
--\end{array}$ & {$[24]$} \\
\hline Asia & $\begin{array}{l}\text { Parupeneus } \\
\text { heptacanthus }\end{array}$ & Escama & 0.46 & 1.2 & {$[25]$} \\
\hline Asia & $\begin{array}{l}\text { Pangasianodon } \\
\text { hypophthalmus } \\
\text { (Pez gato) }\end{array}$ & Piel & 5.1 & 7.7 & {$[26]$} \\
\hline Asia & $\begin{array}{l}\text { Istiophorus } \\
\text { platypterus } \\
\text { (Pez vela) }\end{array}$ & Piel & 5.7 & 2.1 & {$[27]$} \\
\hline Asia & $\begin{array}{l}\text { Lates calcifer } \\
\text { (Barramundi) }\end{array}$ & Escama & 0.38 & 1.06 & [9] \\
\hline Asia & $\begin{array}{l}\text { Ctenopharyngodon } \\
\text { idella } \\
\text { (Carpa herbivora) }\end{array}$ & $\begin{array}{l}\text { Piel } \\
\text { Escama } \\
\text { Aleta }\end{array}$ & $\begin{array}{l}-- \\
-- \\
--\end{array}$ & $\begin{array}{l}-- \\
-- \\
--\end{array}$ & {$[28]$} \\
\hline Asia & $\begin{array}{l}\text { Oreochromis } \\
\text { niloticus } \\
\text { (Tilapia del Nilo) }\end{array}$ & $\begin{array}{l}\text { Escama } \\
\text { Piel }\end{array}$ & $\begin{array}{c}3.2 \\
27.2\end{array}$ & $\begin{array}{l}-- \\
--\end{array}$ & {$[29]$} \\
\hline $\begin{array}{l}\text { América } \\
\text { del Sur }\end{array}$ & $\begin{array}{l}\text { Oreochromis ssp } \\
\text { (Tilapia roja) }\end{array}$ & $\begin{array}{l}\text { Escama } \\
\text { Piel } \\
\text { Hueso }\end{array}$ & $\begin{array}{c}6.5 \\
15.5 \\
5.6 \\
\end{array}$ & $\begin{array}{l}-- \\
-- \\
--\end{array}$ & {$[30]$} \\
\hline Asia & Dover sole & Piel & 19.2 & -- & {$[31]$} \\
\hline
\end{tabular}

REG: Región de origen del pescado.

REND: Rendimiento de la extracción de colágeno.

REF: Referencia.

ASC: Colágeno soluble en ácido.

PSC: Colágeno soluble en pepsina.

\section{DISCUSIÓN}

Como se muestra en la Tabla 1, los diferentes autores que han extraído colágeno a partir de residuos de pescado han encontrado diferencias en los rendimientos de colágeno obtenido. Esto podría deberse, en parte, a las diferentes especies utilizadas, así como a la metodología y cuidado de la temperatura durante la extracción. Se debe tener en cuenta la observación que realiza Nagai ${ }^{[11]}$ respecto a la temperatura de desnaturalización del colágeno respecto al lugar de origen de los peces, pues apunta que el colágeno obtenido de peces que viven en aguas más frías, tiene una temperatura de desnaturalización menor que los que viven en aguas más cálidas. Además, como lo indica Jongjareonrak ${ }^{[5]}$, la cantidad de colágeno aislada también varía con la edad y temporada en la cual se adquirió el pez.

Aún con algunas de las observaciones realizadas por los grupos de investigación sobre los rendimientos obtenidos de colágeno, es importante destacar que la variabilidad del rendimiento es grande con respecto a los datos reportados por Nagai. Para poder obtener rendimientos con una variabilidad mínima es necesario establecer una metodología que sea aplicada para la mayor parte de los peces o incluso hacerlas para aquellos que cumplan la mayor cantidad de características de una población, lo que ayudaría a conocer con mayor exactitud el rendimiento promedio de la extracción de colágeno a partir de los residuos de pescado y de esta manera poder equipararlo e incluso competir con el colágeno obtenido de animales bovinos y porcinos y con ello poder incrementar su aplicación en el área médica y evitando la potencial transmisión de enfermedades que conlleva el uso de colágeno bovino y porcino.

Debido a la literatura consultada referente al material compósito HAp/colágeno a partir de desechos de pescado, se ha observado que existe información limitada sobre este tema, lo cual indica que es un área con gran potencial de investigación para la obtención de materiales que son recuperados de residuos. 


\section{CONCLUSIONES}

La recuperación de materiales, tales como el colágeno y la HAp, a partir de desechos de pescado, es una alternativa importante con impacto positivo para el medio ambiente y para la fabricación de biomateriales de baja toxicidad y mayor biocompatibilidad, con potencial de ser aprovechada con fienes de uso médico, en particular para la regeneración ósea. 


\section{REFERENCIAS}

[1] F. Silver y D. L. Chriistiansen, Biomaterials Science and bioconpatibility Frederick H. Silver. New York, USA: Springer-Velag, 1999.

[2] G. Rh. Owen, M. Dard, y H. Larjava, "Hydoxyapatite/betatricalcium phosphate biphasic ceramics as regenerative material for the repair of complex bone defects”, J. Biomed. Mater. Res. Part B Appl. Biomater., vol. 106, núm. 6, pp. 2493-2512, 2018. doi: 10.1002/jbm.b.34049

[3] J. D. Bronzino, Tissue Engineering and Artificial Organs, Third Edit. New York, USA, 2006.

[4] D. Ulfyana, F. Anugroho, S. H. Sumarlan, y Y. Wibisono, "Bioceramics synthesis of hydroxyapatite from red snapper fish scales biowaste using wet chemical precipitation route", IOP Conf. Ser. Earth Environ. Sci., vol. 131, núm. 1, 2018. doi: $10.1088 / 1755-1315 / 131 / 1 / 012038$

[5] A. Jongjareonrak, S. Benjakul, W. Visessanguan, T. Nagai, y M. Tanaka, "Isolation and characterisation of acid and pepsinsolubilised collagens from the skin of Brownstripe red snapper (Lutjanus vitta)”, Food Chem., vol. 93, núm. 3, pp. 475-484, 2005. doi: 10.1016/j.foodchem.2004.10.026

[6] J. Wang, X. Pei, H. Liu, y D. Zhou, "Extraction and characterization of acid-soluble and pepsin-soluble collagen from skin of loach (Misgurnus anguillicaudatus)", Int. J. Biol. Macromol., vol. 106, pp. 544-550, 2018. doi: 10.1016/j.ijbiomac.2017.08.046

[7] Y. Nomura, H. Sakai, Y. Ishii, y and K. Shirai, "Preparation and Some Properties of Type I Collagen from Fish Scales", Biosci. Biotechnol. Biochem., vol. 60, núm. 12, pp. 2092-2094, 1996. doi: 10.1271/bbb.60.2092

[8] D. Liu, P. Zhou, T. Li, y J. M. Regenstein, "Comparison of acidsoluble collagens from the skins and scales of four carp species", Food Hydrocoll., vol. 41, pp. 290-297, 2014. doi: 10.1016/i.foodhyd.2014.04.030

[9] S. Chuaychan, S. Benjakul, y H. Kishimura, "Characteristics of acidand pepsin-soluble collagens from scale of seabass ( Lates calcarifer )", LWT - Food Sci. Technol., vol. 63, núm. 1, pp. 71-76, 2015. doi: 10.1016/j.lwt.2015.03.002

[10] M. S. H. KIMURA, S. Omura,Y. Ishida, "Molecular Characterization of fibrillar collagen from the body wall of starfish Asterias Amurensis", vol. 104B, núm. 3, pp. 663-668, 1993. doi: 0305-0491/93

[11] T. Nagai, "Isolation of collagen from fish waste material - skin, bone and fins", Food Chem., vol. 68, núm. 3, pp. 277-281, 2000. doi: 10.1016/s0308-8146(99)00188-0

[12] T. Ikoma, H. Kobayashi, J. Tanaka, D. Walsh, y S. Mann, "Physical properties of type I collagen extracted from fish scales of Pagrus major and Oreochromis niloticas.”, Int. J. Biol. Macromol., vol. 32, núm. 3-5, pp. 199-204, 2003. doi: $10.1016 / 50141-8130(03) 00054-0$

[13] M. V. Bhuimbar, P. K. Bhagwat, y P. B. Dandge, "Extraction and characterization of acid soluble collagen from fish waste: Development of collagen-chitosan blend as food packaging film”, J. Environ. Chem. Eng., vol. 7, núm. 2, p. 102983, 2019. doi: 10.1016/j.jece.2019.102983
[14] W. K. Liu, B. S. Liaw, H. K. Chang, Y. F. Wang, y P. Y. Chen, "From Waste to Health: Synthesis of Hydroxyapatite Scaffolds From Fish Scales for Lead Ion Removal”, Jom, vol. 69, núm. 4, pp. 713-718, 2017. doi: 10.1007/s11837-017-2270-5

[15] Y. Wibisono et al., "Synthesis and sinterability of hydroxyapatite from fishery by-products”, J. Korean Ceram. Soc., vol. 55, núm. 6 , pp. 570-575, 2018. doi: 10.4191/kcers.2018.55.6.03

[16] P. Sasmal y H. Begam, "Extraction of Type-I Collagen from Sea Fish and Synthesis of Hap/Collagen Composite", Procedia Mater. Sci., vol. 5, pp. 1136-1140, 2014. doi: 10.1016/j.mspro.2014.07.408

[17] A. B. H. Yoruc y A. K. Aydinoglu, "Synthesis of hydroxyapatite/ collagen (HA/COL) composite powder using a novel precipitation technique”, Acta Phys. Pol. A, vol. 127, núm. 4, pp. 1264-1267, 2015. doi:10.12693/APhysPolA.127.1264

[18] S. Belouafa, A. Bennamara, y A. Abourriche, "Low-cost processing technology for the synthesis of a biocomposite for biomedical applications: A preliminary study”, J. Mater. Environ. Sci., vol. 8, núm. 3, pp. 825-830, 2017.

[19] Y. Chai y M. Tagaya, "Simple preparation of hydroxyapatite nanostructures derived from fish scales”, Mater. Lett., vol. 222, pp. 156-159, 2018. doi: 10.1016/j.matlet.2018.04.009

[20] D. Liu, L. Liang, J. M. Regenstein, y P. Zhou, "Extraction and characterisation of pepsin-solubilised collagen from fins, scales, skins, bones and swim bladders of bighead carp (Hypophthalmichthys nobilis)", Food Chem., vol. 133, núm. 4, pp. 1441-1448, 2012. doi: 10.1016/j.foodchem.2012.02.032

[21] W. Pon-on, P. Suntornsaratoon, N. Charoenphandhu, y J. Thongbunchoo, "Hydroxyapatite from fi sh scale for potential use as bone scaffold or regenerative material", Mater. Sci. Eng. C, vol. 62, pp. 183-189, 2016. doi: 10.1016/j.msec.2016.01.051

[22] T. Nagai, M. Izumi, y M. Ishii, "Fish scale collagen. Preparation and partial characterization”, Int. J. Food Sci. Technol., vol. 39, núm. 3, pp. 239-244, 2004. doi: 10.1111/j.1365-2621.2004.00777.x

[23] I. Bae, K. Osatomi, A. Yoshida, y K. Osako, "Biochemical properties of acid-soluble collagens extracted from the skins of underutilised fishes", Food Chem., vol. 108, pp. 49-54, 2008. doi: 10.1016/j.foodchem.2007.10.039

[24] R. Duan, J. Zhang, X. Du, X. Yao, y K. Konno, "Properties of collagen from skin, scale and bone of carp (Cyprinus carpio)", Food Chem., vol. 112, núm. 3, pp. 702-706, 2009. doi: 10.1016/j.foodchem.2008.06.020

[25] K. Matmaroh, S. Benjakul, T. Prodpran, y A. B. Encarnacion, "Characteristics of acid soluble collagen and pepsin soluble collagen from scale of spotted golden goatfish ( Parupeneus heptacanthus )", Food Chem., vol. 129, núm. 3, pp. 1179-1186, 2011. doi: 10.1016/j.foodchem.2011.05.099

[26] P. Singh, S. Benjakul, S. Maqsood, y H. Kishimura, "Isolation and characterisation of collagen extracted from the skin of striped catfish ( Pangasianodon hypophthalmus )", Food Chem., vol. 124, núm. 1, pp. 97-105, 2011. doi: 10.1016/j.foodchem.2010.05.111

[27] S. Tamilmozhi, A. Veeruraj, y M. Arumugam, "Isolation and characterization of acid and pepsin-solubilized collagen from the skin of sail fi sh ( Istiophorus platypterus )", FRIN, vol. 54, núm. 2, pp. 1499-1505, 2013. doi: 10.1016/i.foodres.2013.10.002 
[28] D. Liu, X. Zhang, T. Li, y H. Yang, "Extraction and characterization of acid- and pepsin-soluble collagens from the scales, skins and swim-bladders of grass carp

(Ctenopharyngodon idella)”, Food Biosci., vol. 9, pp. 68-74, 2015. doi: 10.1016/j.fbio.2014.12.004

[29] J. Chen, L. Li, R. Yi, N. Xu, R. Gao, y B. Hong, "Extraction and characterization of acid-soluble collagen from scales and skin of tilapia ( Oreochromis niloticus )", LWT - Food Sci. Technol., vol. 66, pp. 453-459, 2016. doi: 10.1016/j.lwt.2015.10.070
[30] J. Quintero y J. E. Zapata, “Optimización de la Extracción del Colágeno Soluble en Ácido de Subproductos de Tilapia Roja (Oreochromis spp) mediante un Diseño de Superficie de Respuesta”, Inf. Tecnol., vol. 28, núm. 1, pp. 109-120, 2017. doi: 10.4067/S0718-07642017000100011

[31] G. K. S. Arumugam, D. Sharma, R. M. Balakrishnan, y J. B. P. Ettiyappan, "Extraction, optimization and characterization of collagen from sole fish skin”, Sustain. Chem. Pharm., vol. 9, núm. May, pp. 19-26, 2018. doi: 10.1016/j.scp.2018.04.003 